\title{
Estudo da sorção de césio em solos: avaliação do desempenho em repositório de rejeitos radioativos
}

Amostras de solo provenientes de Minas Gerais (Brasil) foram investigadas quanto à capacidade de sorção de césio (Cs) com vistas a aplicação destes como material de enchimento em um repositório para deposição de rejeitos radioativos de médio e baixo nível de atividade. Foram avaliadas cinco amostras de solo quanto à capacidade máxima de sorção de césio (Q), coeficiente de distribuição (Kd), e outros parâmetros relacionados à sorção: capacidade de troca catiônica, granulometria, composição química e mineralógica. Os parâmetros de sorção ( $Q$ e Kd) foram determinados a partir do ajuste dos dados experimentais, obtidos em ensaios por batelada, a modelos de isotermas de sorção apropriados. Adicionalmente, realizou-se um estudo da correlação entre os parâmetros obtidos pelas isotermas e as demais propriedades dos solos. Evidenciou-se uma forte correlação entre algumas propriedades e a sorção para Cs, fazendo com que estas possam ser utilizadas como indicativos na escolha de solos para serem empregados como camada de recheio em depósitos de rejeito radioativo. Dentre as amostras de solo estudadas, o argilossolo coletado em Conselheiro Pena demonstrou as melhores características de sorção, com valores de Kd de 90,50 mL.g-1 e capacidade máxima de sorção de 18,35 mg.g-1, sendo, portanto, o solo mais promissor para ser utilizado como material de enchimento.

Palavras-chave: Repositório; Rejeito Radioativo; Solos; Sorção de Césio.

\section{Study of cesium sorption in soils: performance assessment in a repository for radioactive waste}

\begin{abstract}
Soil samples of Minas Gerais State (Brazil) were investigated regarding the Cs sorption capacity in order to use them as back filling materials in a disposal facility for low-level radioactive waste. Five samples were evaluated according to their maximum sorption capacity of cesium (Q) and distribution coefficient (Kd), and also other parameters related to sorption: cation exchange capacity, grain size, chemical and mineralogical composition. The sorption parameters ( $Q$ and $K d$ ) were determined from adjustments of experimental data, obtained from batch procedures, to appropriate isotherm models. In addition, a correlation study between isotherm parameters and other soil properties was conducted. It was evidenced a strong correlation between some of these properties and the sorption of Cs, in a way that they can be used as indicators in soil selection to be used as backfilling material in a disposal facility for radioactive waste. Among the samples investigated, the claysoil from Conselheiro Pena presented the best sorption characteristics, with Kd value of $90.50 \mathrm{mg} . \mathrm{g}-1$ and maximum sorption capacity of $18.35 \mathrm{mg} . \mathrm{g}-1$, being the most promising soil to be used as back filling material.
\end{abstract}

Keywords: Repository; Radioactive Waste; Soil; Cesium Sorption.

Topic: Engenharia Nuclear

Reviewed anonymously in the process of blind peer.

Jaqueline Alves de Almeida Calábria

Centro de Desenvolvimento da Tecnologia Nuclear, Brasil http://lattes.cnpq.br/9004358821447092

jaqueline.calabria@cnen.gov.br

\section{Ana Cláudia Queiroz Ladeira}

Centro de Desenvolvimento da Tecnologia Nuclear, Brasil http://lattes.cnpq.br/5014603660790632

acql@cdtn.br

Stela Dalva Santos Cota

Centro de Desenvolvimento da Tecnologia Nuclear, Brasil http://lattes.cnpq.br/3687748297375152

sdsc@cdtn.br
Received: 04/10/2016

Approved: 23/01/2017

Centro de Desenvolvimento da Tecnologia Nuclear, Brasi http://lattes.cnpq.br/1422690785419125 pchr@cdtn.br

Referencing this:

CALÁBRIA, J. A. A.; LADEIRA, A. C. Q.; COTA, S. D. S.; RODRIGUES, P. C. H.. Estudo da sorção de césio em solos: avaliação do desempenho em repositório de rejeitos radioativos. Revista Ibero-Americana de Ciências Ambientais, v.8, n.2, p.190-204, 2017. DOI: http://doi.org/10.6008/SPC2179-6858.2017.002.0016 


\section{INTRODUÇÃO}

A radiação e as substâncias radioativas podem ter muitas aplicações benéficas, dentre as quais, a geração de energia, aplicações em medicina, indústria, meio ambiente e agricultura. Contudo, o uso dos materiais radioativos gera rejeitos que devem ser adequadamente tratados, acondicionados e finalmente dispostos em um repositório.

Um repositório é uma instalação destinada a receber os rejeitos radioativos, depois de tratados e condicionados, confinando-os pelo tempo que se fizer necessário para que decaiam a níveis de radioatividade considerados seguros para o homem e meio ambiente (CNEN, 1989; IAEA, 2001a; IAEA, 2012). Várias alternativas técnicas são possíveis para a deposição de rejeitos radioativos de médio e baixo nível, sendo que a maior parte dos depósitos existentes ou em projeto, incluindo o modelo brasileiro, trata-se de instalações de deposição próximas à superfície (IAEA, 2001a).

Sistemas superficiais de deposição de rejeitos requerem o uso de barreiras de engenharia para controlar a liberação de radionuclídeos, já que as barreiras naturais nesse conceito são virtualmente ausentes. Embora haja diferenças de projeto de uma instalação para outra, é possível identificar em quase todas as construções as seguintes barreiras: o embalado do rejeito contendo a matriz (cimento, betume, polímeros, vidro e cerâmica), a embalagem e os revestimentos; a unidade de deposição contendo a estrutura civil, as camadas de isolamento, camada de recheio e a capa; e a cobertura artificial, contendo uma série de camadas de alta e baixa permeabilidade (IAEA, 2001a).

A segurança global de um sistema de deposição de rejeitos é determinada pela performance individual de cada componente. Especificamente, os materiais de enchimento são empregados com o propósito de preencher vazios entre os embalados e controlar a infiltração de água, a liberação de gases, a sorção e a precipitação de radionuclídeos. Portanto, são propriedades-chaves de um material na avaliação da sua aplicação como material de enchimento, a sua permeabilidade, suas propriedades mecânicas e suas características de sorção. Materiais típicos usados como enchimento são argilas, especialmente as bentonitas, argamassa de cimento e também solos ou combinação de solos (IAEA, 2001a, 2001b).

Em países que possuem plantas nucleares, como o Brasil, o maior volume de rejeito radioativo de médio e baixo nível advém dessas instalações. Os radionuclídeos presentes nesses rejeitos são, em sua maioria, de meia-vida relativamente curta, incluindo ${ }^{137} \mathrm{Cs}$, ${ }^{60} \mathrm{Co},{ }^{90} \mathrm{Sr},{ }^{3} \mathrm{H}$ e ${ }^{55} \mathrm{Fe}$ (IAEA, 2004; IAEA, 2009). No Brasil, o ${ }^{137} \mathrm{Cs}$ apresenta a maior contribuição relativa ao inventário isotópico, com mais de $45 \%$ de participação (AGUIAR, 2006). Em função disto e de sua grande solubilidade, é um dos radionuclídeos de especial interesse para a análise de segurança de uma instalação de deposição de rejeitos radioativos no país.

No meio ambiente, o césio se comporta de maneira muito simples, formando poucos complexos estáveis, de maneira que provavelmente estará presente na forma não complexada, de íons $\mathrm{Cs}^{+}$. Esses íons são fortemente sorvidos por vários minerais, principalmente os argilosos do tipo mica, que fixam o Cs entre suas camadas estruturais. Óxidos de ferro também podem sorver o Cs por complexação, no entanto nesse caso a sorção é reversível. Quando minerais do tipo mica não estão presentes, a sorção de Cs ocorre quase 
que inteiramente por troca catiônica. A sorção de Cs por materiais húmicos é geralmente pequena já que o Cs forma complexos muito fracos com a matéria orgânica. Portanto, parâmetros importantes que possivelmente influenciam os valores de coeficiente de distribuição $-K_{d}$ para esse radionuclídeo, incluem o teor de argilominerais, teor de mica, pH, capacidade de troca catiônica (CTC), área superficial e concentração de Cs em solução (EPA, 1999b).

Este trabalho investiga a sorção de Cs em solos do estado de Minas Gerais (Brasil), com vistas à aplicação destes como camada de recheio em um repositório para rejeitos radioativos de baixa e média atividade. Para isso, inicialmente cinco amostras de solo foram selecionadas a partir de suas características gerais descritas no Sistema Brasileiro de Classificação de Solos - SiBCS e dos dados do Projeto Solos de Minas. Para cada uma dessas amostras, construiu-se isotermas de sorção, às quais ajustou-se modelos matemáticos que forneceram parâmetros cuja avaliação permitiu determinar a qualidade de cada solo como sorvente de Cs. Adicionalmente, avaliou-se a correlação entre algumas propriedades destes solos, tais como a CTC e teor de ferro, e a sorção do Cs.

\section{METODOLOGIA}

\section{Seleção dos tipos de solo e pontos de coleta de amostra}

Para definição dos tipos de solo a serem avaliadas neste estudo, utilizou-se o Mapa de Solos de Minas (FEAM, 2010) e o Sistema Brasileiro Classificação de Solos - SiBCS, definindo-se algumas unidades de mapeamento, baseando-se em sua composição e optando-se por trabalhar com unidades de constituição mais simples, onde apenas um ou poucos tipos de solo fossem observados. Deste modo, selecionou-se as seguintes unidades LVd5, LAd1, LVAd1, NVe1 e PVe4.

De acordo com a Legenda Expandida do Mapa de Solos (FEAM, 2010), a unidade LVd5 é constituída por LATOSSOLO VERMELHO, distrófico, típico, horizonte A moderado, textura argilosa e LATOSSOLO VERMELHO, eutrófico, típico, horizonte A moderado, textura argilosa; ambos fase floresta caducifólia, relevo plano e suave ondulado. A escolha dessa unidade se deu por tratar-se de uma mistura de latossolos vermelhos, em princípio com elevado teor de ferro e boas propriedades de sorção, além disso, a presença de um solo eutrófico, rico em bases trocáveis foi também um critério de escolha. Na contrapartida da unidade LVd5, a unidade LAd1 foi selecionada exatamente por apresentar baixos teores de ferro, para fins de comparação. A unidade LAd1 é composta por LATOSSOLO AMARELO, distrófico, húmico, textura argilosa e LATOSSOLO AMARELO, distrófico, típico, horizonte A proeminente, textura argilosa, ambos fase floresta subcaducifólia e floresta subperenifólia, relevo plano e suave ondulado.

A unidade LVAd1 trata-se de um LATOSSOLO VERMELHO-AMARELO, distrófico, típico, horizonte A moderado, textura argilosa, fase cerrado, relevo plano e suave ondulado, e foi escolhida por acreditar-se que representaria propriedades intermediárias à LVd5 e à LAd1.

As duas unidades restantes são a PVe4, constituída por de 60\% de ARGILOSSOLO VERMELHO, eutrófico, típico, horizonte A moderado, textura média e argilosa, profundo, floresta subperenifólia, relevo 
ondulado e forte ondulado, $20 \%$ de ARGILOSSO VERMELHO-AMARELO distrófico, típico, horizonte A moderado, textura média e argilosa e $20 \%$ de CAMBISSOLO FLÚVICO Tb, eutrófico, típico, horizonte A moderado, textura média, floresta tropical subperenifólia, relevo plano; e a unidade NVe1 um NITOSSOLO VERMELHO, eutrófico, típico, horizonte A chernozêmico/moderado, textura argilosa, fase caatinga hipoxerófila, relevo plano e suave ondulado. Ambas as unidades foram selecionadas em função de seu provável teor elevado de argilominerais, que seria determinante para as propriedades de sorção.

A escolha dos cinco pontos de amostragem foi realizada sobrepondo os pontos do Projeto Solos de Minas (in PEIXOTO, 2013) ao Mapa de Solos de Minas Gerais (FEAM, 2010), e verificando quais pontos estariam localizados em uma área correspondente a cada unidade de mapeamento selecionada previamente. Este tratamento foi realizado no Laboratório de Geoprocessamento do CDTN. Os dados do projeto Solos de Minas, coordenado pela FEAM, consistem da localização exata de aproximadamente 500 pontos de coleta georeferenciados, análises elementares e classificação de acordo com o SiBCS (EMBRAPA, 2006) para cada uma dessas amostras. Estão disponíveis ainda resultados de teor de argila, silte, nitrogênio e potássio orgânicos.

\section{Coleta e preparo das amostras de solo}

A coleta das amostras foi realizada no ponto georeferenciado por um GPS (Global Positioning System) modelo GPSmap 76CSx, fabricado pela GARMIN. O procedimento de coleta seguiu o Manual de Coleta de Solos para Valores de Referência de Qualidade no Estado de Minas Gerais (FEAM, 2013), e consistiu em uma amostragem composta por um ponto central e outros quatro pontos em torno deste, a uma distância de cerca de três a cinco metros na direção dos pontos cardiais (N, S, L, O). A superfície da região de coleta foi limpa de galhos, e vegetação rasteira e foram abertas minitrincheiras de $20 \mathrm{~cm}$ de profundidade e 40 a 50 $\mathrm{cm}$ de diâmetro, em cada um dos cinco pontos. As amostras foram retiradas com o auxílio de uma cavadeira e uma pá. Cada amostra composta foi então seca a temperatura ambiente, em seguida peneirada em peneirador vibratório, marca Mavi-Hude, modelo 6.12/M-03-4, com peneira de malha 20\# Tyler (0,841 mm). A fração retirada foi então desaglutinada fisicamente, e o passante foi britado em um Britador de Rolos, $10^{\prime \prime} 6^{\prime \prime}$, marca DENVER, modelo 1076. Finalmente, homogeneizou-se em pilha longitudinal e quarteou-se em frações de $5 \mathrm{~kg}$, que posteriormente foram subdivididas em frações de $500 \mathrm{~g}$ e $50 \mathrm{~g}$.

\section{Caracterização das amostras de solo}

As amostras de solo foram submetidas às seguintes análises de caracterização: determinação qualitativa dos principais minerais por difração de raios-X pelo método do pó, utilizando o difratômetro Rigaku, $\mathrm{d}$-max ultimate e identificação das fases cristalinas por comparação dos difratogramas de raios-X das amostras com o banco de dados da ICDD- International Center for Diffraction Data/Joint Committee on Powder Difrraction Standard; identificação dos principais elementos por fluorescência de energia dispersiva utilizando o equipamento EDX-720, marca Shimadzu; superfície específica pelo método BET (BrunauerEmmett-Teller), técnica multiple point, equipamento NOVA 2200 Ver. 6.11; determinação da capacidade de 
troca catiônica - CTC pelo método do aceto de amônio, método 5A2a do Soil Survey Laboratory Methods Manual (USDA, 1996); determinação do teor de matéria orgânica através da diferença de massa na perda ao fogo em 550 ㄷ C e 250 드, após removida a umidade; análise granulométrica passando-se o material, previamente deslamado na peneira de $400 \#$ Tyler $(0,037 \mathrm{~mm})$, por uma série de peneiras de aberturas progressivamente menores (20\# Tyler $(0,810 \mathrm{~mm}), 28 \#$ Tyler $(0,590 \mathrm{~mm}), 35 \#$ Tyler $(0,420 \mathrm{~mm}), 48 \#$ Tyler $(0,297 \mathrm{~mm}), 65 \#$ Tyler $(0,210 \mathrm{~mm}), 100 \#$ Tyler $(0,149 \mathrm{~mm}), 150 \#$ Tyler $(0,105 \mathrm{~mm}), 200 \#$ Tyler $(0,074 \mathrm{~mm}), 270 \#$ Tyler $(0,053 \mathrm{~mm}), 325 \#$ Tyler $(0,044 \mathrm{~mm}), 400 \#$ Tyler $(0,037 \mathrm{~mm}))$ e realizando-se a pesagem das frações retidas; determinação da densidade das partículas de solo e pH de acordo com o Manual de Análise de Solos da Embrapa (EMBRAPA, 2011).

\section{Experimentos de sorção em batelada}

Para avaliação da capacidade de sorção de césio pelas amostras de solo construíram-se isotermas de sorção a partir de ensaios por batelada utilizando o método das razões variáveis (EPA, 1992). As razões sólido:líquido utilizadas foram as seguintes: 1:10, 1:20, 1:40, 1:60, 1:80, 1:100, 1:150, 1:200. As massas de solo úmido, considerando-se o teor de umidade, foram medidas para cada razão sólido:líquido e misturadas a $200 \mathrm{~mL}$ de solução contendo $100 \mathrm{mg}^{\mathrm{L}} \mathrm{L}^{-1}$ de Cs, preparada a partir de $\mathrm{CsCl}$ grau analítico. $\mathrm{O} \mathrm{pH}$ dessas misturas foi ajustado para 5,5 $\pm 0,1$, para todas as amostras, utilizando $\mathrm{NaOH}$ ou $\mathrm{HCl}$, conforme necessário. Optou-se por trabalhar nesse valor de $\mathrm{pH}$, pois encontrava-se próximo ao $\mathrm{pH}$ natural dos solos, que variaram de 5,3 a 6,4. As amostras permaneceram em contato com a solução de Cs por 24 horas, após esse período, quando já haviam entrado em equilíbrio, foram separadas por centrifugação seguida de filtração a vácuo em papel de filtro $0,45 \mu \mathrm{m}$. A concentração de Cs na solução resultante foi determinada por espectrometria de emissão atômica, utilizando o espectrômetro AAFS-20, marca Agilent-Varian. Com os resultados das concentrações de Cs no equilíbrio para cada razão sólido:líquido, calculou-se S (massa de Cs adsorvida em $\mathrm{mg} /$ massa de adsorvente em g), e plotou-se versus $C_{\text {eq }}$ (concentração de Cs no equilíbrio em mg. $\mathrm{L}^{-1}$ ), construindo-se assim as curvas de sorção às quais ajustou-se os modelos de isotermas apropriados, conforme descrito no item 2.2.

\section{Análise dos dados}

\section{Ajuste das isotermas de sorção}

Isotermas de sorção são gráficos que correlacionam a massa de soluto retida por unidade de massa de fase sólida, $S(M / M)$, e a concentração do soluto em solução no equilíbrio, $C_{e q}\left(M / L^{3}\right)$, com o objetivo de caracterizar a distribuição de massa entre as fases líquida e sólida (WEBER et al., 1991). Experimentalmente, podem ser obtidas equilibrando uma quantidade conhecida de sorvente com uma solução de concentração conhecida do soluto, enquanto todos os outros parâmetros são mantidos constantes (LANGMUIR, 1997; WEBER \& DIGIANO, 1995). 
O ajuste de modelos matemáticos aos dados experimentais fornece parâmetros a partir dos quais pode-se avaliar as características da sorção em um sistema (WEBER \& DIGIANO, 1995). O modelo de isoterma mais simples é o linear, no qual a retenção do soluto é considerada proporcional à concentração da solução conforme equação 1 :

$$
\mathrm{S}=\mathrm{K}_{\mathrm{d}} \mathrm{C}_{\mathrm{eq}}
$$

A constante de proporcionalidade, $K_{d}$, dada pelo coeficiente angular da reta, é o chamado coeficiente de distribuição e tem dimensões de $\left(\mathrm{L}^{3} / \mathrm{M}\right)$. Dentre as isotermas não lineares, as de Langmuir e Freundlich são as mais comumente empregadas (KINNIBURGH, 1986). Para sistemas sólidos:líquido, a equação de Langmuir é usualmente escrita da seguinte maneira:

$$
\mathrm{S}=\frac{\mathrm{Q}_{\max } \mathrm{K}_{\mathrm{L}} \mathrm{C}_{\mathrm{eq}}}{1+\mathrm{K}_{\mathrm{L}} \mathrm{C}_{\mathrm{eq}}}
$$

O coeficiente $K_{L}\left(M / L^{3}\right)$, é um parâmetro relacionado à entalpia de sorção, ao passo que $Q_{\text {máx }}$ representa a concentração de soluto sorvida correspondente a uma monocamada, comumente definido como capacidade máxima de sorção, e é expresso em unidades de massa do soluto por unidade de massa do sorvente (M/M) (WEBER et al., 1991; LANGMUIR, 1997). Já o modelo de Freundlich não leva em consideração a capacidade de sorção finita em altas concentrações de soluto e pode ser descrito pela equação:

$$
\mathrm{S}=\mathrm{K}_{\mathrm{F}} \mathrm{C}_{\mathrm{eq}}^{\mathrm{n}}
$$

onde $K_{F}\left[\left(L^{3} / M\right)^{n}\right]$ é um parâmetro relacionado à capacidade de sorção para uma concentração específica; $n(-$ ) está relacionado à intensidade da sorção e pode ser entendido como uma medida conjunta da magnitude acumulada e da diversidade de energias associadas a uma reação de sorção em particular (WEBER et al.,1991; WEBER \& DIGIANO, 1995). Quando $n=1$, a isoterma de Freundlich se resume ao modelo linear e $K_{F}=K_{d}$.

Os modelos referenciados anteriormente são considerados modelos clássicos, contudo equações não clássicas podem ser requeridas para ajustar dados relativos à sistemas naturais, como por exemplo, solos, em função da heterogeneidade implícita desses materiais. Dentre as equações não clássicas destacase a equação de Langmuir-Freundlich (WEBER \& DIGIANO, 1995):

$$
\mathrm{S}=\frac{\mathrm{Q}\left(\mathrm{KC}_{\mathrm{eq})}\right)^{\mathrm{n}^{n}}}{1+\left(\mathrm{KC}_{\mathrm{eq}}\right)^{n}}
$$

O parâmetro $Q(M / M)$ é análogo ao $Q_{\text {máx }}$ da equação de Langmuir e indica a capacidade máxima de sorção; o termo $\mathrm{K}\left[\left(\mathrm{L}^{3} / \mathrm{M}\right)^{\mathrm{n}}\right]$ pode ser interpretado como uma componente do $\mathrm{K}_{\mathrm{F}}$ de Freundlich, sendo $\mathrm{K}_{\mathrm{F}}=$ $\mathrm{QK}^{\mathrm{n}^{\prime}} ; \mathrm{n}^{\prime}$ (-) fornece uma indicação da heterogeneidade e caracteriza a forma da distribuição energética na direção de altos valores de energia (WEBER \& DIGIANO, 1995).

Neste trabalho, para o estudo da sorção de Cs pelas amostras de solo, o modelo linear (equação 1) e os não-lineares clássicos, Langmuir (equação 2) e Freundlich (equação 3), foram avaliados quanto ao ajuste dos dados experimentais. $O$ modelo de Langmuir não se mostrou satisfatório, já que o ajuste dos dados ao modelo não convergiu (tentativa de ajuste não apresentada). Por esse motivo, ajustou-se os dados à isoterma de Langmuir-Freundlich (equação 4) que incorpora os conceitos de heterogeneidade em sua dedução. Embora o modelo linear e o modelo de Freundlich fornecessem os parâmetros $K_{d}$, $K_{F}$ e $n$, que possibilitam 
uma análise da qualidade do material como sorvente, foi necessário realizar o ajuste de Langmuir-Freundlich para obtenção do valor de $Q$, já que desejava-se conhecer também a capacidade máxima de sorção das amostras e nem o modelo linear nem o de Freundlich consideram a capacidade de sorção finita em altas concentrações de soluto. $\mathrm{O}$ ajuste aos modelos foi realizado com o auxílio do software Origin 2015.

\section{Correlação entre a sorção e as características dos solos}

A habilidade de sorção de Cs por uma amostra de solo pode ser quase totalmente atribuída à sua capacidade de troca catiônica ou à presença de argilominerais, que aprisionam esse elemento entre as camadas de sua rede cristalina. $\mathrm{O}$ teor de óxidos de ferro também tem relação com a capacidade de sorção, nesse caso o Cs é complexado aos sítios contendo ferro (EPA, 1999b).

No intuito de investigar uma correlação matemática entre a capacidade de sorção e as características dos solos, foram plotados os valores encontrados de $K_{d}$ contra os dados de teor de ferro, CTC e $Q$ para as amostras avaliadas. Como o objetivo era identificar uma relação direta, ajustou-se uma equação de reta e avaliou-se a qualidade do ajuste a partir dos valores dos coeficientes de correlação.

\section{RESULTADOS E DISCUSSÕES}

\section{Seleção dos pontos de coleta de amostra}

$\mathrm{Na}$ tabela 1 são apresentadas resumidamente as características dos pontos de coleta selecionados conforme os critérios descritos anteriormente no item 2.1.1. A figura 1 destaca a localização dos cinco pontos em questão sobre o Mapa de Solos de Minas Gerais.

Tabela 1: Pontos de coleta selecionados e suas principais características.

\begin{tabular}{cccccc}
\hline Ponto & $\begin{array}{c}\text { Ponto Projeto Solos } \\
\text { de Minas }\end{array}$ & $\begin{array}{c}\text { Unidade de } \\
\text { Mapeamento }\end{array}$ & Município & \multicolumn{2}{c}{ Coordenadas Geográficas } \\
\cline { 3 - 6 } & 143 & LVd5 & Janaúba & $-15,69272$ & $-43,46881$ \\
2 & 19 & LAd1 & Taioberas & $-15,81558$ & $-42,09297$ \\
3 & 172 & LVAd1 & Caraí & $-17,15128$ & $-41,50908$ \\
4 & 198 & NVe1 & Salinas & $-16,11183$ & $-42,31719$ \\
5 & 157 & PVe4 & Conselheiro Pena & $-19,13250$ & $-41,57247$ \\
\hline
\end{tabular}

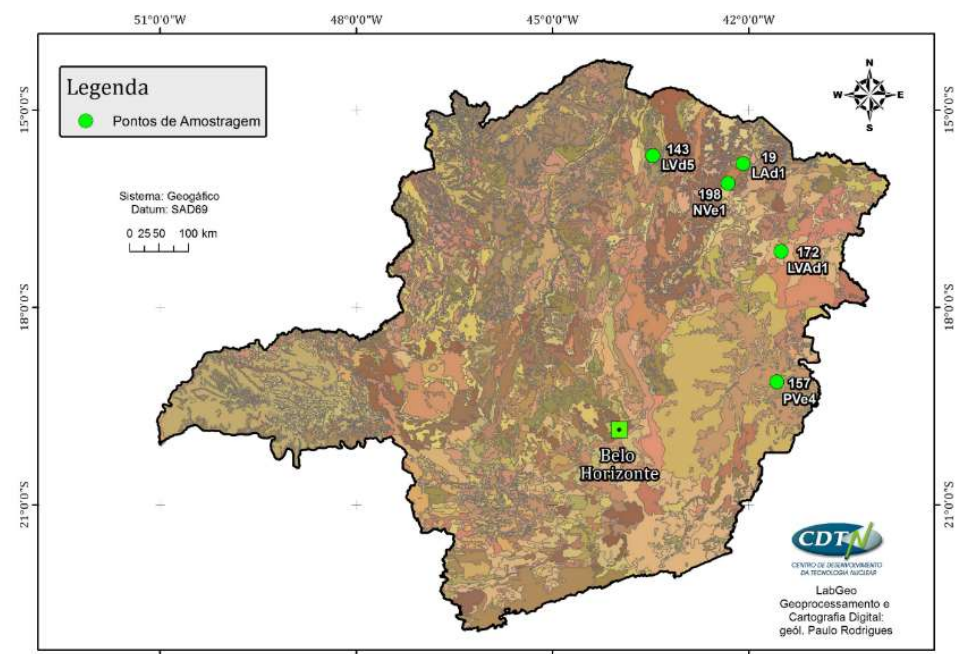

Figura 1: Localização dos cinco pontos de amostragem selecionados referentes a cada uma das unidades de mapeamento: LVd5, LAd1, LVAd1, NVe1, PVe4, sobre o Mapa de Solos de Minas Gerais (FEAM, 2010). 


\section{Caracterização das amostras de solo}

A tabela 2 apresenta os resultados de caracterização físico-química, química e mineralógica das amostras de solo em estudo. Todas as amostras são ligeiramente ácidas, sendo a mais ácida delas a de Taioberas e a que apresenta o pH mais próximo de neutro, a de Janaúba. Os valores de densidade não foram muito discrepantes variando entre 2,50 e $2,79 \mathrm{~g} . \mathrm{cm}^{-3}$ e foram utilizados para auxiliar na medida de volume dos solos.

Nota-se que as amostras de Janaúba e Conselheiro Pena, com 76,60\% e 69,28\% da fração abaixo de 400\# Tyler, respectivamente, apresentam maior percentual de finos em relação as demais, destoando principalmente das amostras de Taioberas, $28,10 \%$ e Caraí, 39,82\%. A fração fina é a mais relevante do ponto de vista da sorção de contaminantes, pois além de normalmente comportar os minerais argilosos, o fenômeno de sorção também pode ser correlacionado com a área superficial.

Os solos com maiores áreas superficiais foram Janaúba $\left(45,25 \mathrm{~m}^{2} \cdot \mathrm{g}^{-1}\right)$ e Conselheiro Pena $\left(40,75 \mathrm{~m}^{2} \cdot \mathrm{g}\right.$ $\left.{ }^{1}\right)$, respectivamente, com superfícies específicas aproximadamente duas vezes maior do que Salinas (21,09 $\left.\mathrm{m}^{2} \cdot \mathrm{g}^{-1}\right)$ e três vezes Caraí $\left(13,05 \mathrm{~m}^{2} \cdot \mathrm{g}^{-1}\right)$ e Taioberas $\left(13,67 \mathrm{~m}^{2} \cdot \mathrm{g}^{-1}\right)$, que apresentaram valores praticamente iguais. Esses resultados estão concordantes com a análise granulométrica, já que as amostras com maiores valores de área superficial, foram as mesmas que apresentaram os maiores percentuais das frações finas (retidas na peneira de 400\# Tyler $(0,037 \mathrm{~mm})$ ).

Os maiores valores de capacidade de troca catiônica foram encontrados para as amostras de Conselheiro Pena $(10,08 \mathrm{meq} / 100 \mathrm{~g})$ e Janaúba $(8,75 \mathrm{meq} / 100 \mathrm{~g})$, respectivamente, embora ainda sejam valores baixos se comparados com a bentonita comercial ( $77 \mathrm{meq} / 100 \mathrm{~g})$ e demais argilas (SHEPPARD \& THIBAULT, 1990). A troca catiônica é um dos principais mecanismos de retenção de césio em meios minerais, portanto é uma propriedade relevante na avaliação da sorção para esse radionuclídeo (EPA, 1999a).

Quanto à composição química, os cátions como $\mathrm{Na}^{+}, \mathrm{K}^{+}, \mathrm{Ca}^{2+}$ e $\mathrm{Mg}^{2+}$, são cátions trocáveis, e a sua concentração nas amostras tem correlação com a capacidade de troca catiônica das mesmas, e por consequência com a sorção de Cs. A amostra de Conselheiro Pena, que apresentou o maior valor de troca catiônica (10,08 meq/100g), exibiu também maior valor de concentração dos cátions, $\mathrm{K}^{+}, \mathrm{Ca}^{2+}$ e $\mathrm{Mg}^{2+}(2,89 \%)$, seguida por Salinas (1,17\%), Janaúba (0,70\%), Caraí $(0,55 \%)$ e Taioberas $(0,20 \%)$.

O ferro desempenha também papel importante na sorção de césio. Grupos das extremidades, como - $\mathrm{Fe}-\mathrm{OH}_{2}$, ao serem ionizados geram sítios negativos, que embora não permanentes, podem atrair cátions eletrostaticamente. Deste modo, embora a troca catiônica seja considerada o principal mecanismo de retenção para o césio, ele pode também ser sorvido por óxidos de ferro, sendo este, inclusive, o mecanismo dominante de sorção em minerais que possuem baixa ou nenhuma carga permanente, e que, portanto, apresentam baixos valores de CTC (McBRIDE, 1994; EPA, 1999a). Os maiores teores de ferro foram encontrados para as amostras de Conselheiro Pena e Janaúba, seguida por Salinas, Caraí e Taioberas. Para as amostras de Conselheiro Pena e Janaúba, o ferro está presente principalmente na goethita e na hematita, mas pode também ser encontrado na rede da muscovita e rutilo em menor proporção. 
Tabela 2. Caracterização das amostras de solo.

\begin{tabular}{|c|c|c|c|c|c|c|}
\hline \multicolumn{7}{|c|}{ Caracterização das Amostras de Solo } \\
\hline & & $\begin{array}{l}\text { Taioberas } \\
\text { LAd1 }\end{array}$ & $\begin{array}{r}\text { Caraí } \\
\text { LVAd1 }\end{array}$ & $\begin{array}{l}\text { Conselheiro Pena } \\
\text { PVe4 }\end{array}$ & $\begin{array}{l}\text { Salinas } \\
\text { NVe1 }\end{array}$ & $\begin{array}{l}\text { Janaúba } \\
\text { LVd5 }\end{array}$ \\
\hline \multicolumn{7}{|c|}{ Análises Físico-Química } \\
\hline & Densidade $\left(\mathrm{g} \cdot \mathrm{cm}^{-3}\right)$ & 2,50 & 2,79 & 2,53 & 2,67 & 2,50 \\
\hline & $\mathrm{pH}$ & 4,40 & 5,31 & 5,77 & 6,01 & 6,43 \\
\hline & CTC $($ meq/100g) & 5,95 & 5,02 & 10,08 & 6,05 & 8,75 \\
\hline & \% da fração retida em $0,037 \mathrm{~mm}$ & 28,10 & 39,82 & 69,28 & 53,46 & 76,00 \\
\hline & Área Superficial $\left(\mathrm{m}^{2} \cdot \mathrm{g}^{-1}\right)$ & 13,67 & 13,05 & 40,75 & 21,09 & 45,25 \\
\hline \multicolumn{7}{|c|}{ Análise Química } \\
\hline \multirow{14}{*}{ 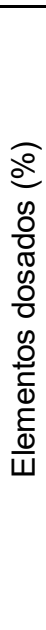 } & $\mathrm{MgO}$ & 0,15 & $<0,01$ & 0,69 & 0,12 & 0,08 \\
\hline & $\mathrm{Al}_{2} \mathrm{O}_{3}$ & 26,00 & 25,00 & 26,75 & 21,85 & 28,65 \\
\hline & $\mathrm{SiO}_{2}$ & 61,00 & 61,00 & 48,15 & 60,30 & 44,70 \\
\hline & $\mathrm{K}_{2} \mathrm{O}$ & 0,04 & 0,55 & 1,88 & 0,85 & 0,42 \\
\hline & $\mathrm{CaO}$ & $<0,10$ & 0,05 & 0,32 & 0,20 & 0,20 \\
\hline & $\mathrm{Fe}_{2} \mathrm{O}_{3}$ & 3,10 & 4,56 & 9,82 & 5,81 & 9,05 \\
\hline & $\mathrm{Na}_{2} \mathrm{O}$ & $<1,00$ & $<1,00$ & $<1,00$ & $<1,00$ & $<1,00$ \\
\hline & $\mathrm{Ti}$ & 0,46 & 0,48 & 0,67 & 0,51 & 0,81 \\
\hline & $\mathrm{Mn}$ & 0,02 & 0,02 & 0,08 & 0,11 & 0,13 \\
\hline & $\mathrm{Ba}$ & 0,13 & 0,14 & 0,19 & 0,15 & 0,23 \\
\hline & $S$ & 0,08 & 0,08 & 0,07 & 0,08 & 0,07 \\
\hline & $\mathrm{Zr}$ & 0,02 & 0,06 & 0,03 & 0,02 & 0,04 \\
\hline & Co, V, Ni, Cr, Cu, As, Rb, Th e U & $<0,10$ & $<0,10$ & $<0,10$ & $<0,10$ & $<0,10$ \\
\hline & Matéria Orgânica & 8,91 & 8,20 & 11,40 & 8,50 & 15,80 \\
\hline \multicolumn{7}{|c|}{ Difração de Raios-X } \\
\hline \multicolumn{7}{|c|}{ Mineral Identificado } \\
\hline & Predominante $(>60 \%)$ & - & Quartzo & - & - & - \\
\hline & Maior $(<50 \%)$ & $\begin{array}{l}\text { Quartzo } \\
\text { Caulinita }\end{array}$ & 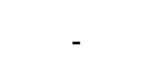 & $\begin{array}{l}\text { Caulinita } \\
\text { Quartzo }\end{array}$ & $\begin{array}{l}\text { Quartzo } \\
\text { Caulinita }\end{array}$ & $\begin{array}{l}\text { Caulinita } \\
\text { Quartzo }\end{array}$ \\
\hline & Menor $(<15 \%)$ & $\begin{array}{l}\text { Gibsita } \\
\text { Haloisita }\end{array}$ & $\begin{array}{c}\text { Haloisita } \\
\text { Caulinita } \\
\text { Muscovita }\end{array}$ & $\begin{array}{l}\text { Haloisita Goethita } \\
\text { Muscovita }\end{array}$ & $\begin{array}{l}\text { Muscovita } \\
\text { Hematita }\end{array}$ & $\begin{array}{l}\text { Haloisita } \\
\text { Hematita }\end{array}$ \\
\hline & Minoritário (<3\%) & - & - & - & - & Rutilo \\
\hline \multicolumn{7}{|c|}{$\begin{array}{c}\text { Caulinita: } \mathrm{Al}_{2} \mathrm{Si}_{2} \mathrm{O}_{5}(\mathrm{OH})_{4} ; \text { Gibsita: } \mathrm{Al}(\mathrm{OH})_{3} ; \text { Goethita: } \mathrm{FeO}(\mathrm{OH}) ; \text { Haloisita: } \mathrm{Al}_{2} \mathrm{Si}_{2} \mathrm{O}_{5}(\mathrm{OH})_{4} ; \mathrm{Hematita:} \mathrm{Fe}_{2} \mathrm{O}_{3} \text {; } \\
\text { Muscovita: }\left(\mathrm{K}, \mathrm{NH}_{4}, \mathrm{Na}\right) \mathrm{Al}_{2}(\mathrm{Si}, \mathrm{Al})_{4} \mathrm{O}_{10}(\mathrm{OH})_{2}, \text { Quartzo: } \mathrm{SiO}_{2} \text {, Rutilo: } \mathrm{TiO}_{2} \text {. }\end{array}$} \\
\hline
\end{tabular}

Argilominerais tendem a fixar o Cs entre as suas camadas estruturais (EPA, 1999a). Dentre os minerais encontrados nos solos, i.e., quartzo, muscovita, gibbsita, hematita, goetita, caulinita e haloisita, os dois últimos são caracterizados como argilominerais, embora a capacidade de retenção de íons de ambos seja muito baixa; quase inexistente para a caulinita. A estrutura ideal da caulinita não possui carga $(<0,01)$ e por esse motivo, suas camadas não têm tendência a se separar, a menos que o ambiente seja altamente polar; assim, possui baixas área superficial e capacidade de troca catiônica. A haloisita possui a mesma estrutura da caulinita, no entanto apresenta uma camada de moléculas de água entre as folhas. Quando aquecida, se converte em caulinita de forma irreversível (McBRIDE, 1994). Na tabela 2, o mineral referenciado primeiramente em uma mesma linha, possui teor maior que os demais; assim, a amostra de Conselheiro Pena, apresenta o maior teor aproximado de argilominerais (caulinita e haloisita) e o maior teor de minerais do tipo mica (muscovita). 


\section{Isotermas de sorção para o Cs}

Os dados de sorção para o Cs, ajustados ao modelo de Langmuir-Freundlich (equação 4), são apresentadas na figura 2(a-d). Esse ajuste permitiu obter o valor de $Q$ para cada uma das amostras, já que os dados não se ajustaram apropriadamente ao modelo convencional de Langmuir, que não convergiu. Os dados para a amostra de Taioberas não se ajustaram ao modelo Langmuir-Freundlich, ou a qualquer um dos modelos não-lineares clássicos e, portanto, não estão representados. A Figura 2(e) apresenta os dados experimentais de todas as amostras sem o ajuste ao modelo Langmuir-Freundlich, apenas para melhor comparação do desempenho dos solos. A tabela 3 apresenta os parâmetros obtidos para o ajuste à equação de Langmuir-Freundlich para cada uma das amostras, bem como os valores dos coeficientes de correlação $\left(R^{2}\right)$.

O parâmetro Q obtido a partir do ajuste de Langmuir-Freundlich (tabela 3) diz respeito a capacidade máxima de sorção e é um indicativo da qualidade do material como sorvente. Dentre as amostras avaliadas, Conselheiro Pena apresentou o maior valor de $Q\left(18,3472 \mathrm{mg} \cdot \mathrm{g}^{-1}\right)$ para Cs. Embora os valores de Q para todas as amostras possam ser considerados como superdimensionados, uma vez que o patamar de sorção não foi atingido (figura 2), em termos comparativos, existe evidência do melhor desempenho da amostra de Conselheiro Pena (figura 2(e)). Nota-se ainda, que mesmo não sendo possível determinar o valor do parâmetro Q para a amostra de Taiobeiras, avaliando-se a figura 2(e), é possível perceber que a tendência é de que esse parâmetro, correspondente à saturação da sorção, para essa amostra, possua o menor valor entre todos.

Tabela 3: Ajustes às isotermas de Langmuir-Freundlich relativas à sorção de Cs.

\begin{tabular}{|c|c|c|c|c|c|}
\hline Amostra & $\begin{array}{l}\text { Equação de Langmuir-Freundlich } \\
\qquad S=Q \cdot K \cdot C^{n^{\prime}} /\left(1+K \cdot C^{n^{\prime}}\right)\end{array}$ & $\mathrm{R}^{2}$ & $Q\left(m g \cdot g^{-1}\right)$ & $K\left(L \cdot g^{-1}\right)^{n}$ & $\mathrm{n}^{\prime}$ \\
\hline $\begin{array}{l}\text { Conselheiro } \\
\text { Pena }\end{array}$ & $S=\left(18,3472^{*} 0,0188^{*} C^{N}(0,7343)\right) /\left(1+0,0188^{*} C^{\wedge}(0,7343)\right)$ & 0,9936 & 18,3472 & 0,0188 & 0,7343 \\
\hline Janaúba & $S=\left(8,0316^{*} 0,0111^{*} C^{N}(0,9308)\right) /\left(1+0,0111^{*} C^{N}(0,9308)\right)$ & 0,9932 & 8,0316 & 0,0111 & 0,9308 \\
\hline Salinas & $S=\left(2,1218^{*} 0,0019^{*} C^{\wedge}(1,6874)\right) /\left(1+0,0019^{*} C^{\wedge}(1,6874)\right)$ & 0,9542 & 2,1218 & 0,0019 & 1,6874 \\
\hline Caraí & $S=\left(2,6671^{\star} 0,0123^{*} C^{\wedge}(0,9387)\right) /\left(1+0,0123^{*} C^{\wedge}(0,9387)\right)$ & 0,9715 & 2,6671 & 0,0123 & 0,9387 \\
\hline Taioberas & NÃO AJUSTA & - & - & - & - \\
\hline
\end{tabular}

A tabela 4 apresenta as equações de Freundlich e linear para cada uma das amostras, bem como os valores dos parâmetros extraídos a partir dessas equações. A figura 3 apresenta o ajuste dos dados aos modelos linear (equação 1), e de Freundlich (equação 3), para as amostras de solo.

Os valores de $\mathrm{K}_{d}$ e $\mathrm{K}_{\mathrm{F}}$ (tabela 4), parâmetros obtidos pelo ajuste linear e de Freundlich respectivamente, estão relacionados a extensão da sorção, podendo ser utilizados para comparar diferentes materiais. Dentre as amostras avaliadas, Conselheiro Pena apresentou os maiores valores de $K_{d}\left(90,50 \mathrm{~mL}^{-1}{ }^{-1}\right), K_{F}(437,00$ (mg.g 
$\left.\left.{ }^{1}\right) \cdot\left(\mathrm{mL} \cdot \mathrm{g}^{-1}\right)^{\mathrm{n}}\right)$ e $\mathrm{Q}\left(18,35 \mathrm{mg} \cdot \mathrm{g}^{-1}\right)$ (tabela 3), demonstrando que existe uma relação entre estes parâmetros. Deste modo, Conselheiro Pena mostrou-se o solo mais apropriado para sorção de Cs dentre os analisados. $O$ solo de Janaúba, apresentando valores de $K_{d}=45,90 \mathrm{~mL} \cdot \mathrm{g}^{-1}, \mathrm{~K}_{\mathrm{F}}=149,10\left(\mathrm{mg} \cdot \mathrm{g}^{1}\right) \cdot\left(\mathrm{mL} \cdot \mathrm{g}^{-1}\right)^{\mathrm{n}}$ ) e $\mathrm{Q}=8,03 \mathrm{mg} \cdot \mathrm{g}^{-1}$, foi considerado o segundo melhor solo para sorção de Cs dentre os estudados. Observa-se ainda, pela figura 3, que para todas as amostras as isotermas de Freundlich são levemente convexas, como demonstrado pelos valores de $\mathrm{n}<1$ (tabela 4), indicando sorção favorável embora baixa. O processo de sorção favorável é aquele no qual a capacidade de retenção aumenta com a quantidade de soluto, fornecendo uma isoterma convexa (WEBER \& DIGIANO, 1995).
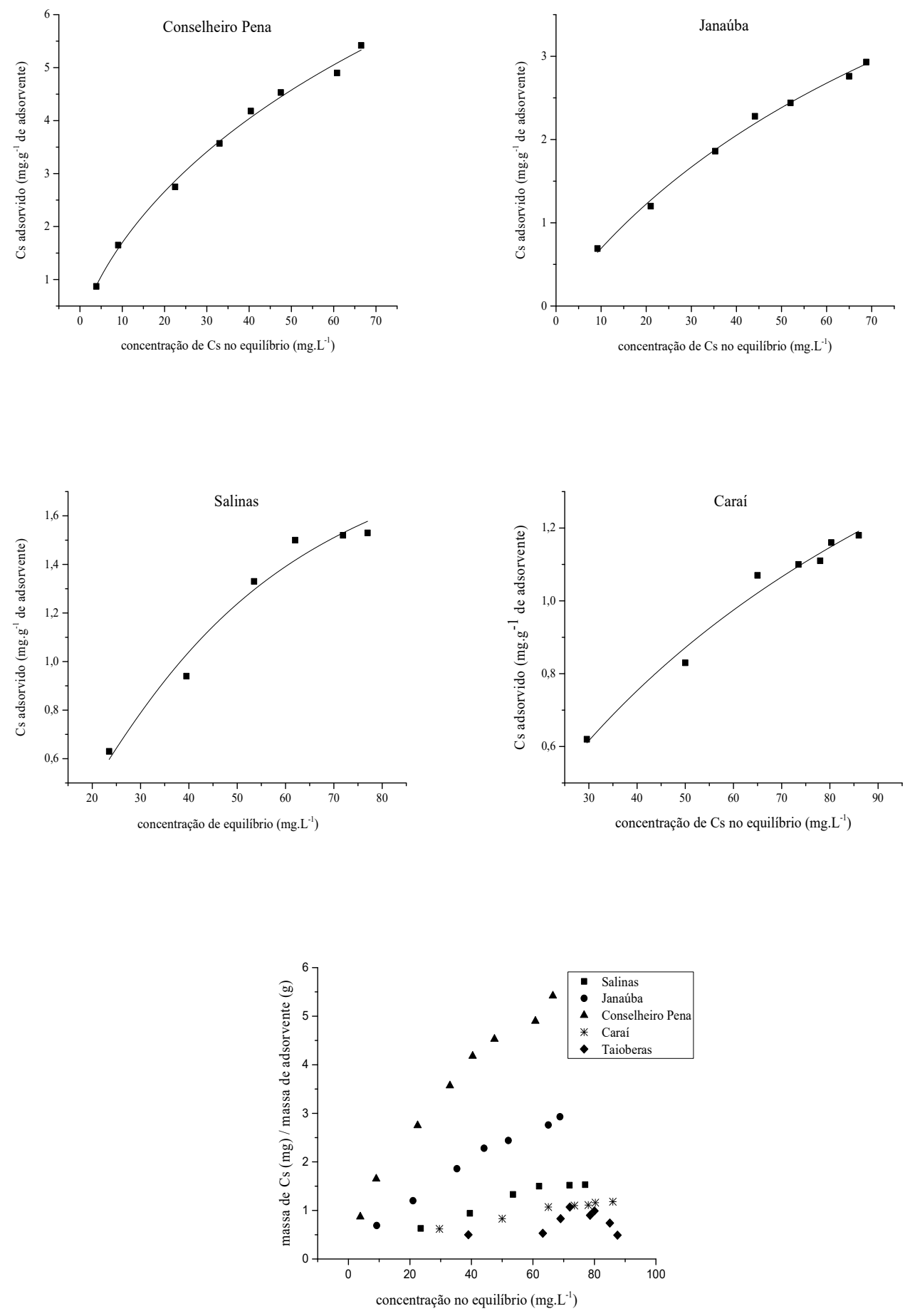

Figura 2: Isotermas de Langmuir-Freundlich para sorção de Cs para cada amostra de solo (a-d), (e) dados apresentados em conjunto. $\mathrm{T}=25 \circ \mathrm{C}$, tempo de equilíbrio $=24 \mathrm{~h}, \mathrm{pH}=5,5 \pm 0,1$. 
Aldaba et al. (2010) estudaram 9 solos representativos do território espanhol, no intuito de comparar os valores de $K_{d}$ para Cs obtidos em ensaios por batelada com valores estimados através de experimentos de difusão. As características dos solos apresentadas no estudo são insuficientes para avaliar se há alguma amostra que se assemelhe aos solos investigados neste trabalho. No entanto, tomando-se apenas o pH como critério, analisando-se os valores de $K_{d}$ encontrados por Aldaba et al. (2010) para as amostras de solos ácidos (4 entre 9), tem-se os seguintes resultados: 0,55 mL.g ${ }^{-1}, 8 \mathrm{~mL} \cdot \mathrm{g}^{-1}, 13 \mathrm{~mL} \cdot \mathrm{g}^{-1}$ e $481 \mathrm{~mL} . \mathrm{g}^{-1}$. Desconsiderando-se os extremos, os resultados são comparáveis (da mesma ordem de grandeza) àqueles obtidos neste estudo, ainda que mais baixos.

Tabela 4: Ajustes às isotermas linear e de Freundlich.

\begin{tabular}{|c|c|c|c|c|c|c|c|c|c|}
\hline \multirow{3}{*}{ Amostra } & \multicolumn{4}{|c|}{ Linear } & \multicolumn{5}{|c|}{ Freundlich } \\
\hline & \multirow{2}{*}{$\begin{array}{c}\text { Equação } \\
S=K_{d} \cdot C_{e q}\end{array}$} & \multirow[t]{2}{*}{$\mathrm{R}^{2}$} & \multicolumn{2}{|c|}{$K_{d}$} & \multirow{2}{*}{$\begin{array}{l}\text { Equação } \\
\mathrm{S}=\mathrm{K}_{\mathrm{F}} \cdot \mathrm{C}_{\mathrm{eq}}{ }^{\mathrm{n}}\end{array}$} & \multirow[t]{2}{*}{$\mathrm{R}^{2}$} & \multicolumn{2}{|c|}{$\mathrm{K}_{\mathrm{F}}$} & \multirow[t]{2}{*}{$\mathrm{n}$} \\
\hline & & & $\left(\mathrm{L} \cdot \mathrm{g}^{-1}\right)$ & $\left(\mathrm{mL} \cdot \mathrm{g}^{-1}\right)$ & & & $\left(\mathrm{mg} \cdot \mathrm{g}^{-1}\right) \cdot\left(\mathrm{L} \cdot \mathrm{g}^{-1}\right)^{\mathrm{n}}$ & $\left(m g \cdot g^{-1}\right) \cdot\left(m L \cdot g^{-1}\right)^{n}$ & \\
\hline $\begin{array}{c}\text { Conselheiro } \\
\text { Pena }\end{array}$ & $S=0,0905 C_{e q}$ & 0,9716 & 0,0905 & 90,5 & $S=0,4370 C^{0,5990}$ & 0,993 & 0,4370 & 437,00 & 0,5990 \\
\hline Janaúba & $S=0,0459 C_{e q}$ & 0,9873 & 0,0459 & 45,9 & $S=0,1491 C^{0,7050}$ & 0,992 & 0,1491 & 149,10 & 0,7050 \\
\hline Salinas & $\mathrm{S}=0,0222 \mathrm{C}_{\mathrm{eq}}$ & 0,9892 & 0,0222 & 22,2 & $S=0,0661 C^{0,7369}$ & 0,936 & 0,0661 & 66,10 & 0,7369 \\
\hline Caraí & $S=0,0149 C_{e q}$ & 0,9899 & 0,0149 & 14,9 & $S=0,0775 C^{0,6155}$ & 0,975 & 0,0775 & 77,50 & 0,6155 \\
\hline
\end{tabular}
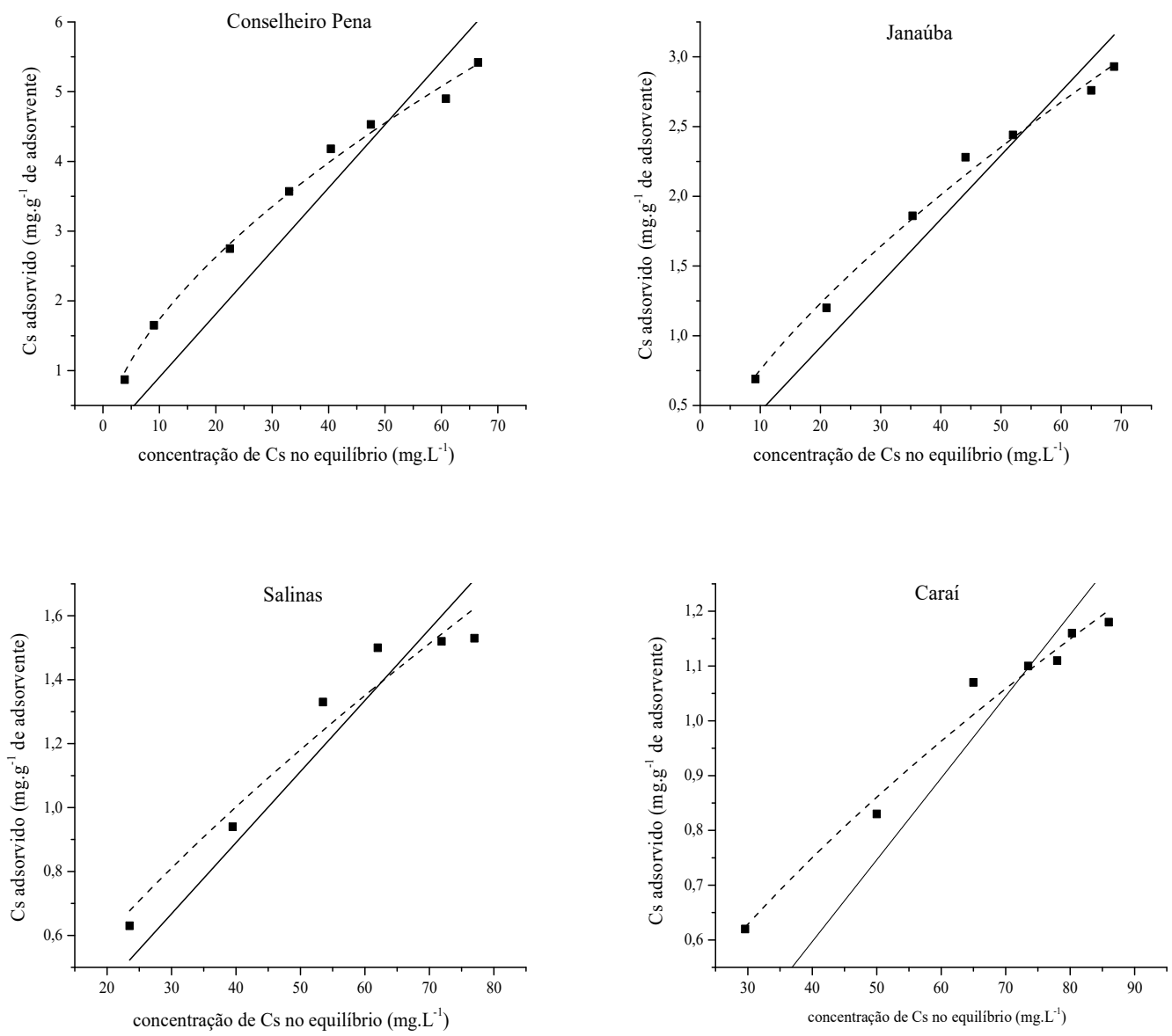

Figura 3: Isotermas de Freundlich (tracejado) e linear (sólido), para sorção de Cs, para cada amostra de solo. T=25ㄷ, tempo de equilíbrio=24 horas, $\mathrm{pH}=5,5 \pm 0,1$. 


\section{Correlação entre as propriedades de sorção e características dos solos}

Os dados de teor de ferro, CTC e $Q$, para os solos em estudo, versus os valores de $K_{d}$ determinados são apresentados na figura 4. Como não foi possível obter ajuste satisfatório aos modelos de isoterma para a amostra de Taioberas, ela foi excluída deste estudo de correlação.

(a)
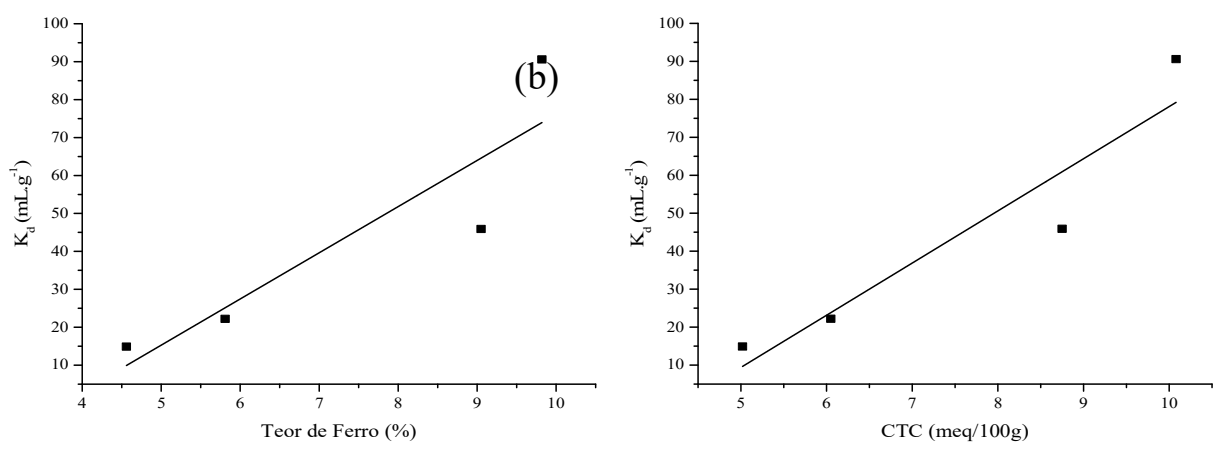

(c)

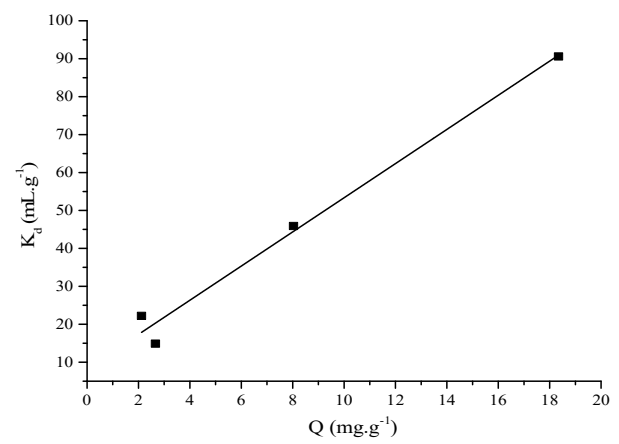

Figura 4: (a) $K_{d} \times$ Teor de ferro, (b) $K_{d} \times C T C$ e (c) $K_{d} \times Q$, para as amostras de solo (excluindo-se a amostra de Taiobeiras).

As correlações obtidas entre os valores de $K_{d}$ e teor de ferro, CTC e $Q$, apresentaram coeficientes de correlação $\left(R^{2}\right)$ de $0,8114,0,8897$ e 0,9857, respectivamente, demonstrando que para as amostras estudadas há uma relação direta entre os parâmetros: teor de ferro, CTC e $Q$, e $K_{d}$. As expressões matemáticas que definem esses ajustes são apresentadas pelas equações 5, 6 e 7.

$$
\begin{aligned}
& K_{d}\left(m L . g^{-1}\right)=12,1700 \text { Teor de ferro }(\%)-45,5860 \\
& K_{d}\left(m L . g^{-1}\right)=13,7420 \text { CTC (meq/100g) }-59,3210 \\
& K_{d}\left(m L . g^{-1}\right)=4,5024 Q\left(m g \cdot g^{-1}\right)+8,3276
\end{aligned}
$$

Uma vez que a análise mineralógica é aproximada, não é apropriado estabelecer uma relação matemática entre os teores dos minerais que interferem na sorção e os resultados de $K_{d}$, no entanto, qualitativamente nota-se que aquela amostra cujo teor de minerais do tipo mica (muscovita) somado ao teor de argilominerais (caulinita e haloisita) foi o maior, i.e, Conselheiro Pena, apresentou também o maior valor de $K_{d}$. Muscovita, caulinita e haloisita, embora não sejam considerados sorvedores excepcionais, quando comparados com os demais minerais identificados nas amostras de solo (ex. quartzo) apresentam uma maior capacidade de sorção. 
No que diz respeito ao teor de finos e à área superficial, ainda que exista uma relação entre esses parâmetros e a sorção, uma vez que os maiores valores de $K_{d}$ e $Q$ estão associados às amostras com maior percentual de finos e áreas superficiais, Janaúba e Conselheiro Pena, essa relação não é linear, já que o ajuste proposto com os dados obtidos apresentou baixo coeficiente de correlação, $R^{2}$ em torno de 0,5000 . Janaúba apresenta o maior teor de finos e a maior área superficial, no entanto o seu valor de $\mathrm{K}_{d}, 45,90 \mathrm{~mL} . \mathrm{g}^{-1}$, é aproximadamente a metade do valor de $\mathrm{K}_{\mathrm{d}}$ de Conselheiro Pena, $90,50 \mathrm{~mL} \cdot \mathrm{g}^{-1}$. Com base nos dados, pode-se inferir que, embora a área superficial seja importante para a sorção, ela não pode ser tomada como um parâmetro que individualmente afete o processo, mas que associado a outros fatores, como teor de ferro e CTC, contribua para melhorar as propriedades de sorção.

O teor de matéria orgânica em princípio não influencia significativamente a sorção de Cs (EPA,1999a), contudo as amostras com maiores valores de $K_{d}$ foram aquelas cujos valores de matéria orgânica foram os maiores, Conselheiro Pena e Janaúba, respectivamente. Observa-se ainda que as amostras com maiores teores de sílica, Caraí e Salinas, apresentaram as mais pobres características de sorção.

\section{CONCLUSÕES}

As previsões iniciais, feitas a partir das características de cada solo, de que o argilossolo (Conselheiro Pena) e o latossolo vermelho (Janaúba) apresentariam melhores resultados de sorção para Cs, foram confirmadas neste estudo, assim como a baixa capacidade de sorção para o latossolo amarelo (Taioberas). A amostra de argilosolo de Conselheiro Pena apresentou os melhores resultados quanto à sorção de Cs, tanto no que se refere ao valor da capacidade de carregamento $\left(Q=18,35 \mathrm{mg} \cdot \mathrm{g}^{-1}\right)$, quanto ao coeficiente de distribuição $\left(K_{d}=90,50 \mathrm{~mL} \cdot \mathrm{g}^{-1}\right)$ e $\mathrm{K}_{\mathrm{F}}\left(437,00\left(\mathrm{mg} \cdot \mathrm{g}^{-1}\right) \cdot\left(\mathrm{mL} \cdot \mathrm{g}^{-1}\right)^{\mathrm{n}}\right)$. As características dos solos que demonstraram a partir de um ajuste matemático estar diretamente relacionadas à sorção de Cs foram o teor de ferro e a capacidade de troca catiônica. $\mathrm{O}$ argilossolo de Conselheiro Pena apresentou, dentre as amostras investigadas, os maiores valores para esses parâmetros e ainda o maior teor de argilominerais (caulinita e haloisita) e o segundo maior teor de finos, características que influenciam positivamente a sorção de Cs.

Entretanto, pode-se dizer que em comparação com argilas comerciais $\left(K_{d}=1900 \mathrm{~mL} \cdot \mathrm{g}^{-1}\right)$ especialmente as bentonitas, comumente empregadas como material de enchimento em repositórios, o valor de $K_{d}$ para o solo de Conselheiro Pena ainda é baixo. Por outro lado, o estudo de solos locais se justifica plenamente devido a sua disponibilidade e custo, tendo em vista a grande quantidade desses materiais normalmente utilizada em um repositório de rejeitos radioativos.

O emprego deste argilossolo como enchimento em um repositório, fica condicionado à avaliação de segurança da instalação, e à avaliação do desempenho das barreiras de engenharia, que, em função das características da instalação, podem tanto indicar que o solo puro é adequado ao uso, como demonstrar que o mesmo deverá ser combinado com outros materiais para aplicação. Em qualquer dos casos, os resultados obtidos para os valores de $K_{d}$ neste estudo, bem como sua relação com as características dos solos, podem 
ser empregados como guias na escolha de materiais locais que possam ser utilizados no projeto e construção do repositório brasileiro.

Também os resultados obtidos neste estudo podem ser utilizados como suporte na caracterização e seleção de locais candidatos a abrigarem depósitos de rejeitos radioativos no Brasil, principalmente a partir da correlação encontrada entre as propriedades do solo e suas características de sorção.

\section{REFERÊNCIAS}

AGUIAR, L. A.. Avaliação de risco de um repositório próximo à superfície na fase pós-fechamento em cenário de liberação de radionuclídeos por infiltração de água. Tese (Doutorado em Ciências em Engenharia Nuclear) Universidade Federal do Rio de Janeiro, Rio de Janeiro, 2006.

ALDABA, D.; RIGOL, A.; VIDAL, M.. Diffusion experiments for estimating radiocesium and radiostrontium sorption in unsaturated soils from Spain: Comparison with batch sorption data. Journal of Hazardous Materials. v.181, p.1072-1079, 2010. DOI: http://doi.org/10.1016/j.jhazmat.2010.05.124

CNEN. Comissão Nacional de Energia Nuclear. CNEN-NE6.06: Seleção e escolha de locais para depósitos de rejeitos radioativos. Rio de Janeiro: CNEN, 1989.

EMBRAPA. Empresa Brasileira de Pesquisa Agropecuária. Sistema brasileiro de classificação de solos. 2 ed. Rio de Janeiro: Embrapa Solos, 2006.

EMBRAPA. Empresa Brasileira de Pesquisa Agropecuária. Manual de métodos de análise de solo. 2 ed. Rio de Janeiro: Embrapa Solos, 2011.

EPA. Environmental Protection Agency. EPA/530/SW-006-F: Batch-type procedures for estimating soil adsorption of chemicals. Washington, 1992.

EPA. Environmental Protection Agency. EPA 402-R-99-004-A: Understanding variation in partition coefficient, $\mathrm{Kd}$, values. Washington, 1999a.

EPA. Environmental Protection Agency. EPA 402-R-99-004-B: Understanding variation in partition coefficient, $\mathrm{Kd}$, values. Washington, 1999b.

FEAM. Fundação Estadual do Meio Ambiente. Mapa de solos do Estado de Minas Gerais: legenda expandida. Belo Horizonte, 2010.

FEAM. Fundação Estadual do Meio Ambiente. Manual de coleta de solos para valores de referência de qualidade no Estado de Minas Gerais. Belo Horizonte, 2013.

IAEA. International Atomic Energy Agency. Technical considerations in the design of near surface disposal facilities for radioactive waste. Vienna: IAEA, 2001a. IAEATECDOC-1256.

IAEA. International Atomic Energy Agency. Performance of engineered barrier materials in near surface disposal facilities for radioactive waste. Vienna: IAEA, 2001b. IAEATECDOC-1255.

IAEA. International Atomic Energy Agency. Long term behaviour of low and intermediate level waste packages under repository conditions. Vienna: IAEA, 2004. IAEATECDOC-1397.

IAEA. International Atomic Energy Agency. Classification of radioactive waste. Vienna: IAEA, 2009. IAEA Safety Standards Series No. GSG-1.

IAEA. International Atomic Energy Agency. The safety case and safety assessment for the disposal of radioactive waste. Vienna: IAEA, 2012. IAEA Specific Safety Guide No. SSG-23.

KINNIBURGH, D. G.. General Purpose Adsorption Isotherms. Environmental Science \& Technology, v.20, n.9, p.895-904, 1986.

LANGMUIR, D.. Aqueous environmental chemistry. Upper Saddle River: Prentice-Hall, 1997.

McBRIDE, M.. Environmnental chemistry of soils. New York: Oxford University Press, 1994.

PEIXOTO, C. M.. Determinação dos valores de referência de qualidade de solo para U e Th no Estado de Minas Gerais. Dissertação (Mestrado em Ciência e Tecnologia das Radiações, Minerais e Materiais) - Centro de Desenvolvimento da Tecnologia Nuclear, Belo Horizonte, 2013.

SHEPPARD, M. I., THIBAULT, D. H.. Default soil solid/liquid partition coefficients, $K_{d} S$, for four major soil types: a compendium. Health Physics, v.50, p.471-482, 1990.

USDA. United States Department of Agriculture. Soil Survey Laboratory Methods Manual: Soil survey investigation report №42. Version 3.0. Washington, 1996.

WEBER, W. J.; McGINLEY, P. M.; KATZ, L. E.. Sorption phenomena in surface systems: concepts, models and effects on contaminant fate and transport. Water Research, v. 25 , n.5, p.499-528, 1991. DOI: http://doi.org/10.1016/0043-1354(91)90125-A

WEBER, W. J.; DIGIANO, F. A.. Process dynamics in environmental systems. John Wiley \& Sons, 1995. 